\title{
Consenso Brasileiro de Espondiloartropatias: Outras Espondiloartropatias Diagnóstico e Tratamento - Primeira Revisão
}

\section{First Update on the Brazilian Consensus for the Diagnosis and Treatment of Spondyloarthropathies: Other Spondyloarthropathies}

Percival D. Sampaio-Barros ${ }^{(1)}$, Valderílio Feijó Azevedo( ${ }^{(2)}$, Rubens Bonfiglioli( ${ }^{(3)}$, Wesley R. Campos ${ }^{(4)}$, Sueli Coelho da Silva Carneiro ${ }^{(5)}$, Marco Antonio P. Carvalho ${ }^{(6)}$, Célio Roberto Gonçalves ${ }^{(7)}$, Maria Odete E. Hilário ${ }^{(8)}$, Mauro W. Keiserman ${ }^{(9)}$, Nocy H. Leite ${ }^{(10)}$, Karen Mallmann ${ }^{(11)}$, Eduardo de Souza Meirelles ${ }^{(12)}$, Walber P. Vieira ${ }^{(13)}$, Antonio Carlos Ximenes ${ }^{(14)}$

Descrição do método e coleta de evidências: Reunião consensual para elaboração do texto com inclusão das citações bibliográficas, numa colaboração de reumatologistas com as especialidades de reumatologia pediátrica, dermatologia, coloproctologia e oftalmologia. Foram convidados 10 reumatologistas responsáveis pelo ambulatório de espondiloartropatias em suas instituições (ou seus representantes); cada participante foi convidado a fazer uma análise crítica, utilizando o conceito da medicina baseada em evidências, de um aspecto diferente dentro do espectro do diagnóstico e tratamento das espondiloartropatias; foi convidado um especialista em cada área para fazer a mesma análise crítica nos tópicos espondiloartropatias juvenis (reumatologista pediátrico), psoríase (dermatologista), doenças inflamatórias intestinais (coloproctologista) e uveite anterior (oftalmologista). Após a análise do Consenso Brasileiro de Espondiloartropatias (2004) como texto-base de apoio para as discussões, todos os especialistas se reuniram no período entre 4 e 5 de maio de 2007, a fim de discutir individualmente cada um dos 14 tópicos elaborados e estabelecer um consenso baseado em evidências, a partir do qual o coordenador redigiu o texto do consenso, submetido à apreciação de todos os participantes para a realização dos ajustes finais.

\footnotetext{
1. Assistente-doutor da Disciplina de Reumatologia do Departamento de Clínica Médica da Faculdade de Ciências Médicas da Universidade Estadual de Campinas (FCM-UNICAMP). Presidente da Comissão de Espondiloartropatias da Sociedade Brasileira de Reumatologia (SBR).

2. Professor Assistente da Disciplina de Reumatologia da Universidade Federal do Paraná (UFPR). Mestre em Medicina Interna

3. Professor Assistente da Disciplina de Reumatologia da Pontifícia Universidade Católica de Campinas (PUCCAMP).

4. Professor Adjunto, Doutor em Oftalmologia da Universidade Federal de Minas Gerais (UFMG).

5. Professora Adjunta da Faculdade de Ciências Médicas da Universidade Estadual do Rio de Janeiro (UERJ) e Professora do Programa de Pós-Graduação em Medicina da Universidade Federal do Rio de Janeiro (UFRJ)

6. Professor Adjunto, Doutor de Reumatologia do Departamento do Aparelho Locomotor da Universidade Federal de Minas Gerais (UFMG).

7. Professor Doutor-Assistente e Coordenador da Unidade de Espondiloartropatias da Disciplina de Reumatologia da Faculdade de Medicina da Universidade de São Paulo (FMUSP).

8. Professora Associada e Responsável pelo Setor de Reumatologia Pediátrica da Universidade Federal de São Paulo (UNIFESP).

9. Professor Regente da Disciplina de Reumatologia da Faculdade de Medicina da Pontifícia Universidade Católica do Rio Grande do Sul (PUC-RS).

10. Professor Titular de Reumatologia da Faculdade de Medicina Souza Marques, Rio de Janeiro - RJ.

11. Professora da Disciplina de Coloproctologia da Fundação Faculdade Federal de Ciências Médicas de Porto Alegre (FFFCMPA).

12. Assistente-Doutor e Chefe do Grupo de Reumatologia do Instituto de Ortopedia e Traumatologia da FMUSP.

13. Chefe do Serviço de Reumatologia e Coordenador da Residência Médica do Hospital Geral de Fortaleza.

14. Chefe do Departamento de Medicina Interna do Hospital Geral de Goiânia. Doutor em Reumatologia pela FMUSP

Conflitos de interesse: (1) Recebimento de honorários por conferência ou palestra, bem como financiamento para realização de pesquisa, organização de atividade de ensino ou comparecimento a simpósios e congressos: Abbott, Actelion, Bristol-Myers-Squibb, Mantecorp, Merck Sharp E Dohme, Pfizer, Roche e Wyeth; (2) Auxílio para viagens e recebimento de honorários por palestras: Abbott e Mantecorp; (5) Auxílio para viagens e comparecimento a simpósios e congressos: Mantecorp e Wyeth; (7) Auxílio para participação em congresso: Abbott; (9) Recebimento de honorários por conferência ou palestra, bem como financiamento para realização de pesquisa, organização de atividade de ensino ou comparecimento a simpósios e congressos: Abbott e Bristol-Myers-Squibb; ( 12 ) Recebimento de honorários por conferência ou palestra: Abbott e Mantecorp; (14) Recebimento de honorários por conferência ou palestra, bem como financiamento para realização de pesquisa, organização de atividade de ensino ou comparecimento a simpósios e congressos: Abbott, Bristol-Myers-Squibb, Mantecorp, Merck Sharp E Dohme, Pfizer, Roche e Wyeth. (3), (4), (6), (8), (10), (11) e (13) declararam não ter conflito de interesse.

Endereço para correspondência: Dr. Percival D. Sampaio-Barros. Av. Brigadeiro Luiz Antonio, 2466 - conjs. 93-94, CEP 01402-000. São Paulo, SP, Brasil, e-mail: sbre@terra.com.br
} 
Objetivo: Oferecer informações sobre a otimização do diagnóstico e do tratamento das diferentes espondiloartropatias.

Grau de recomendação e força de evidência científica:

A: Grandes ensaios clínicos aleatorizados e meta-análises.

B: Estudos clínicos e observacionais bem desenhados.

C: Relatos e séries de casos clínicos.

D: Publicações baseadas em consensos e opiniões de especialistas.

\section{INTRODUÇÃO}

O conceito das espondiloartropatias soronegativas foi estabelecido em 1974, quando os pesquisadores ingleses Moll e Wright propuseram que se englobassem dentro de um mesmo conjunto algumas doenças até então consideradas completamente distintas entre si, mas que na verdade apresentavam diversas características comuns. Tais características englobavam aspectos clínicos (dor axial inflamatória, associada à artrite, predominante em grandes articulações de membros inferiores, e entesopatias periféricas), radiológicos (sacroiliíte) e laboratoriais (soronegatividade para o fator reumatóide, pois, até a década de 1970, alguns pesquisadores consideravam a espondilite anquilosante como o componente axial da artrite reumatóide), em indivíduos com predisposição genética (ligada ao antígeno de histocompatibilidade HLA-B27). Este conjunto incluiu a espondilite anquilosante, a artrite psoriásica, a artrite reativa e a síndrome de Reiter, e as artropatias enteropáticas

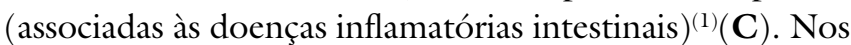
últimos 20 anos, em virtude do crescente número de pacientes que apresentam um diagnóstico provável, mas que não conseguem preencher os critérios diagnósticos para uma doença definida dentro do grupo, criou-se o conceito das espondiloartropatias indiferenciadas. Recentemente, especialistas internacionais propuseram a mudança de nome para espondiloartropatias, suprimindo o termo soronegativas $^{(2)}(\mathbf{C})$.

\section{CONSIDERAÇÕES GERAIS SOBRE A ABOR- DAGEM DIAGNÓSTICA DE PACIENTES COM ESPONDILOARTROPATIAS}

Cada uma das doenças definidas dentro do grupo (espondilite anquilosante, artrite psoriásica, artrite reativa e artrite enteropática) tem seus próprios critérios diagnósticos. No entanto, existem muitos casos ditos indiferenciados ou incompletos. Na tentativa de conseguir englobar todo este amplo e heterogêneo grupo de pacientes, propuseram-se vários critérios classificatórios. Analisando-os, observa-se que os critérios do Grupo Europen de Estudo das Espondiloartropatias ${ }^{(3)}(\mathbf{D})$ (Tabela 1) são os mais utilizados na prática clínica diária, sendo facilmente lembrados e aplicados; para ser incluído dentro do grupo é necessário preencher pelo menos um critério maior e um critério menor.

TABELA 1

CRITÉRIOS CLASSIFICATÓRIOS DE ESPONDILOARTROPATIAS Grupo Europeu de Estudo das Espondiloartropatias (ESSG), 1991

Dor axial inflamatória ou $\begin{aligned} & \text { Sinovite assimétrica predominante em } \\ & \text { membros inferiores }\end{aligned}$
e
Pelo menos um dos seguintes critérios:

História familiar positiva (espondilite anquilosante, psoríase, uveíte anterior, doença inflamatória intestinal)

Psoríase cutânea

Doença inflamatória intestinal

Uretrite ou diarréia aguda até 4 semanas precedendo a artrite

Dor em nádegas alternante

Entesopatia (inserção do tendão de Aquiles ou fáscia plantar)

Sacroiliíte (bilateral graus 2 a 4 ou unilateral graus 3 ou 4 )

Extraída da referência 3.

Sensibilidade: $86 \%$; especificidade: $87 \%$.

\section{CONSIDERAÇÕES GERAIS SOBRE A ABOR- DAGEM TERAPÊUTICA DE PACIENTES COM ESPONDILOARTROPATIAS}

As doenças crônicas, como as espondiloartropatias, na maioria das vezes causam um sentimento de desamparo e inutilidade mais assustador do que a doença em si. Por isso, deve-se sempre informar o paciente, de forma realista, sobre as perspectivas prognósticas de sua doença, perspectivas essas que são freqüentemente otimistas, desde que haja uma abordagem global de atendimento. O paciente deve estar absolutamente ciente de que sua participação - especialmente no estar bem consigo mesmo, apesar da doença - será um ponto de partida fundamental para o sucesso terapêutico. Deve-se realizar o seguimento dos pacientes e o tratamento de forma individual, caso a caso, já que a história natural da doença pode cursar com flutuações durante seu curso evolutivo. O médico deve considerar o impacto da doença para o indivíduo dentro de sua comunidade, com as devidas implicações no seu ambiente familiar e de trabalho, 
além dos aspectos sociais e financeiros. Apesar de inexistir, até o momento, um tratamento específico para a cura, deve-se ressaltar que os recursos terapêuticos disponíveis possibilitam, de um modo geral, um adequado controle da doença. Um ponto fundamental para tais conquistas está centrado numa boa relação médico-paciente; quando necessário, o médico assistente deve indicar auxílio psicoterápico e/ou uso de drogas antidepressivas.

\section{SÍNDROME DE REITER / ARTRITE REATIVA}

O termo Sindrome de Reiter, proposto em 1942 por Bauer e Engleman, atualmente se restringe aos casos caracterizados pela tríade uretrite, artrite e conjuntivite, que ocorre após infecção geniturinária ou gastrointestinal estando incluído no grupo das artrites reativas. $\mathrm{O}$ moderno conceito de artrite reativa dentro do grupo das espondiloartropatias requer a presença de uma artrite asséptica associada à evidência de infecção precedente ${ }^{(4)}(\mathbf{D})$.

A prevalência da artrite reativa é de 30 a 40 casos por 100.000 adultos, enquanto que sua incidência anual é de 4,6 / 100.000 nos casos associados à Chlamydia e de 5 / 100.000 nos casos associados à infecção intestinal ${ }^{(5)}(\mathbf{D})$. Entretanto, os números podem ser mais altos; há estimativas de que 1 a $3 \%$ dos pacientes com uretrite por $\operatorname{Chlamydia}^{(6)}(\mathbf{D})$ e 6 a $30 \%$ dos pacientes com infecção enterobacteriana ${ }^{(7)}(\mathbf{D})$ desenvolverão artrite.

$\mathrm{O}$ agente infeccioso representa, no indivíduo geneticamente predisposto, o "fator gatilho" para o desenvolvimento da doença. Na etiologia pós-disentérica, predominam as bactérias enteropatogênicas, como Shigella (flexneri, sonnei), Salmonella (typhimurium, enteritidis), Yersinia (enterocolitica, pseudotuberculosis) e Campylobacter (jejuni); já nos casos de etiologia geniturinária, predomina a Chlamydia trachomatis $^{(5)}(\mathbf{D})$.

Numerosos fatores imunológicos concorrerão para o desencadeamento da doença. Com relação à interação agente infeccioso-hospedeiro, ocorre invasão das células mucosas, com replicação intracelular do agente infeccioso; há uma eliminação antigênica deficiente, permitindo que antígenos bacterianos se liguem a macrófagos e monócitos (com função de células apresentadoras de antígenos) e atinjam a circulação, ativando as imunidades humoral e celular. Em muitos casos, observa-se uma diminuição dos níveis de citocinas Thl, indispensáveis para a eliminação de inúmeros microrganismos. Em alguns casos de doença crônica, pode-se detectar determinantes antigênicos (geralmente lipopolissacárides de membrana, de Chlamydia,
Shigella ou Salmonella) na biópsia de membrana sinovial; a persistência destes determinantes antigênicos, mesmo na ausência do microrganismo vivo, poderia ser responsável pela perpetuação da doença ${ }^{(8)}(\mathbf{D})$. Já o achado de RNA de Chlamydia, em combinação com demonstração de partículas semelhantes à Chlamydia e/ou antígenos de Chlamydia na membrana sinovial de pacientes com Síndrome de Reiter sugere que células bacterianas viáveis estão presentes naquele tecido ${ }^{(9)}(\mathbf{D})$.

As manifestações clínicas costumam se iniciar de uma a quatro semanas após infecção; na etiologia entérica é comum quadro diarréico auto-limitado, por vezes disentérico, enquanto que, na etiologia geniturinária, ocorre uretrite com sintomatologia leve. Dentro da tríade característica da doença, a primeira manifestação costuma ser a uretrite, freqüentemente serosa, com descarga matinal, oligosintomática; seu diagnóstico é difícil nas mulheres, podendo evoluir para salpingite aguda ou crônica, enquanto nos homens pode cursar com prostatite, muitas vezes assintomática. A conjuntivite costuma ser leve, de resolução espontânea, durando 7 a 10 dias, sem deixar seqüelas. $\mathrm{O}$ quadro articular costuma ser caracterizado por uma oligoartrite assimétrica, recorrente, de predomínio em grandes articulações de membros inferiores, sendo comum a presença de volumosos derrames articulares recorrentes em joelhos, em pacientes com doença ativa. Na evolução do quadro articular, é bastante frequente a presença de entesites na inserção do tendão de Aquiles e da fáscia plantar, também cursando com tenossinovites em dedos de pés e mãos (os chamados "dedos em salsicha").

Acometimento axial (espondilite) pode aparecer em cerca de $20 \%$ dos casos. Comprometimento visceral é pouco freqüente. Pode apresentar evolução como surto único (10\% a $20 \%)$, crises recorrentes $(40 \%$ a $60 \%$ ) ou cronificar (20\% a $30 \%)$. A pesquisa do HLA-B27, positiva em $50 \%$ a $80 \%$ dos casos, não é importante como diagnóstico, mas sim como determinante de prognóstico da doença ${ }^{(10)}(\mathbf{D})$.

$\mathrm{Na}$ infầncia, a maioria dos casos de artrite reativa ocorre em meninos de 8 a 12 anos, estando associadas predominantemente às infecções entéricas. Já nos adolescentes, a artrite reativa posterior à infecção genital por Chlamydia ocorre com maior freqüência.

\section{DIAGNóstico}

Não há critérios diagnósticos validados para a artrite reativa. Entre os critérios propostos, os mais utilizados são os do III Workshop Internacional de Artrite Reativa 
(1996), que define a doença como uma oligoartrite assimétrica predominante em membros inferiores, associada à evidência de infecção prévia ou atual através de história ou exame físico ou por exame laboratorial, excluindo-se outra doença reumática ${ }^{(11)}(\mathbf{D})$ (Tabela 2). O diagnóstico laboratorial da infecção bacteriana pode ser feito por sorologia, pesquisando anticorpos, e por detecção direta do organismo infeccioso na porta de entrada ou na $\operatorname{articulação~}^{(5)}(\mathbf{D})$.

TABELA 2

CRITÉRIOS DIAGNÓSTICOS DE ARTRITES REATIVAS Kingsley e SiePer, 1996

\begin{tabular}{l} 
Artrite assimétrica predominante em membros inferiores \\
Evidência de infecção precedente \\
\hline História de diarréia ou uretrite num período de 4 semanas precedendo a artrite \\
Cultura de fezes positiva \\
Detecção de $C$. trachomatis no exame de urina ou no swab urogenital \\
Anticorpos contra lipopolissacárides ou outros antígenos específicos anti- \\
Yersinia ou anti-Salmonella de subtipo IgG-IgA ou IgG-IgM \\
Anticorpos contra C. trachomatis \\
Detecção de DNA de $C$. trachomatis na articulação por reação de cadeia de \\
polimerase \\
\hline Extraída da referência 11.
\end{tabular}

\section{TRATAMENTO}

$\mathrm{Na}$ fase articular aguda da doença, inicia-se o tratamento com $A I N H$, em doses convencionais; o tratamento deverá durar enquanto houver atividade articular da doença, não havendo nenhum AINH comprovadamente superior aos demais $^{(12)}(\mathbf{D})$. Os corticosteróides costumam ser utilizados em casos de oligoartrite ou entesopatia persistentes não responsivas aos AINHs (prednisona, na dose de 5 a $10 \mathrm{mg}$ / dia, ou equivalente) ou monoartrite persistente (triancinolona hexacetonide intra-articular $)^{(12)}(\mathbf{D})$.

Nos casos que evoluem para doença articular crônica, predominantemente periférica, pode-se utilizar a sulfasalazina $^{(13)}(\mathbf{A})$ e o metotrexato ${ }^{(14)}(\mathbf{C})$, nas doses convencionais. Em pacientes com artrite reativa, nos quais se considera iniciar o uso de droga imunossupressora, devese pesquisar HIV, pois alguns pacientes aidéticos podem apresentar quadro clínico de Síndrome de Reiter como manifestação inicial e, se tratados com imunossupressores, podem desencadear a doença em toda sua expressão.

Quanto aos agentes biológicos anti-TNF $\alpha$, existem relatos esporádicos de boa resposta ao uso de infliximabe ${ }^{(15)}$ $(\mathbf{C})$ e etanercepte ${ }^{(16)}(\mathbf{C})$, mas sua indicação não está bem estabelecida nas artrites reativas.
Deve-se sempre considerar a administração de antibióticos quando a infecção está presente, sendo a sua eficácia dependente do agente causal da moléstia. Nas infecções entéricas causadas por Salmonella, Shigella e Campylobacter, só há indicação de tratamento na presença de infecção ativa; nestes casos, pode-se usar a ciprofloxacina $(500 \mathrm{mg}$, via oral, duas vezes por dia) ou sulfametoxazol-trimetoprin, durante 7 a $10 \operatorname{dias}^{(17)}(\mathbf{D})$.

Em pacientes com infecção ativa do trato geniturinário por Chlamydia, deve-se administrar tratamento antibiótico com tetraciclina ( $500 \mathrm{mg}$, via oral, três vezes por dia) ou derivados (doxiciclina ou minociclina) durante 14 dias $^{(17)}$ (D); é imprescindível o tratamento dos parceiros, para se evitar reinfestações. Como a infecção urogenital por Chlamydia é muitas vezes assintomática, ou cursa com sintomas leves (geralmente não referidos pelos pacientes), e sendo a Chlamydia um importante fator causal de prostatite crônica (assintomática em 50\% dos casos) e salpingite crônica (causa não hormonal freqüente de infertilidade feminina), é prudente que se investiguem pacientes com diagnóstico de artrite reativa sem fator causal definido.

Há controvérsias quanto à eficácia do uso crônico de antibióticos nas artrites reativas por Chlamydia, tanto com relação à ciprofloxacina [ contrário $^{(18)}(\mathbf{A})$ e favorável ${ }^{(19)}(\mathbf{A})$ ] quanto à doxiciclina $\left[\right.$ contrário $^{(20)}(\mathbf{A})$ e favorável $\left.{ }^{(21)}(\mathbf{A})\right]$.

\section{ARTRITES ENTEROPÁTICAS}

As doenças inflamatórias intestinais (DII) podem acometer de 2 a 20 indivíduos em cada 200.000 pessoas; já o acometimento articular pode afetar $2 \%$ a $26 \%$ dos pacientes com DII. Classicamente, podemos observar manifestações articulares associadas à retocolite ulcerativa e à doença de Crohn, e, em menor proporção, à doença de Whipple, doença celíaca e cirurgia de bypass intestinal ${ }^{(22)}(\mathbf{D})$.

Os acometimentos articulares nas DII. podem ser subdivididos em oligoartrite periférica, poliartrite periférica e espondilite enteropática. A oligoartrite periférica acomete preferencialmente grandes articulações de membros inferiores, associada a entesopatias periféricas (notadamente em inserção de tendão aquileano e fáscia plantar); não tem predomínio de sexo ou antígeno de histocompatibilidade específico, e sua evolução está invariavelmente associada à atividade da doença intestinal; lesões cutâneas, tipo eritema nodoso, podem ocorrer em $10 \%$ a $25 \%$ destes pacientes. Poliartrite periférica, geralmente não deformante, pode ocorrer na doença de Whipple e após cirurgia de bypass intestinal; quadro semelhante, porém potencialmente mais 
agressivo e deformante, pode ocorrer em pacientes com doença de Crohn; também não apresentam associação com HLA específico, e sua evolução costuma ser independente do acometimento intestinal. A espondilite enteropática pode acometer $2 \%$ a $12 \%$ dos pacientes com retocolite ulcerativa e doença de Crohn; predomina no sexo masculino (2-3:1), e $50 \%$ a $75 \%$ desses pacientes apresentam HLA-B27 positivo; o quadro clínico e radiológico da espondilite enteropática é semelhante ao observado na espondilite anquilosante, e sua evolução costuma ser independente do quadro intestinal $^{(23)}(\mathbf{D})$.

\section{TRATAMENTO}

Em virtude da possibilidade de causarem ulceração e/ ou sangramento intestinais, confundindo o médico sobre a atividade intestinal da doença, os antiinflamatórios não hormonais não são prescritos de rotina em pacientes com artrite enteropática $^{(22)}(\mathbf{D})$. É preferível o uso de corticosteróides em baixas doses (prednisona $5-10 \mathrm{mg} / \mathrm{dia}$, ou equivalente) para o alívio da artrite ou entesopatia periféricas; indica-se o uso de doses elevadas (até $1 \mathrm{mg} / \mathrm{kg} / \mathrm{dia}$ de prednisona, ou equivalente) nos casos de inflamação intestinal importante ${ }^{(24)}(\mathbf{D})$.

Embora os derivados da 5-ASA, a mesalazina e a sulfasalazina, sejam considerados drogas de primeira linha para o tratamento dos surtos de atividade da doença, recente metanálise apresentou resultados desapontadores ${ }^{(25)}(\mathbf{A})$. Nos casos mais graves de recorrência da doença de Crohn ou doença intestinal extensa ou formação de múltiplas fístulas, o uso do metotrexato(26) $(\mathbf{A})$, da azatioprina ${ }^{(27)}$ (B), do infliximabe ${ }^{(28)}(\mathbf{A})$ e do adalimumabe $^{(29)}(\mathbf{B})$ pode ser indicado. Não existem estudos específicos de avaliação da resposta do componente articular ao tratamento com drogas de base para as doenças inflamatórias intestinais; geralmente a melhora intestinal está associada à melhora do componente periférico da artrite enteropática.

\section{ESPONDILOARTROPATIAS INDIFERENCIADAS}

As espondiloartropatias indiferenciadas (EI) englobam um grupo de pacientes que apresentam características clínicas e/ou radiológicas sugestivas de uma espondiloartropatia, mas que não preenchem os critérios diagnósticos de nenhuma das doenças definidas dentro do grupo. Muitas vezes representam um diagnóstico provisório, englobando um conjunto bastante heterogêneo de pacientes, que pode variar de um estágio precoce ou uma forma abortiva ou frustra de uma espondiloartropatia definida ${ }^{(30)}(\mathbf{D})$.

Estudos controlados de longo prazo indicaram que um paciente com dor inflamatória em sacroilíacas pode demorar até 14 anos para desenvolver uma sacroiliíte radiológica, permitindo então ser feito o diagnóstico de $\operatorname{EA}^{(31)}(\mathbf{C})$. A presença do HLA-B27 parece representar um fator prognóstico de evolução para uma doença definida ${ }^{(32)}(\mathbf{C})$.

\section{DIAGNóstico}

Os pacientes que preenchem os critérios de classificação para espondiloartropatias do Grupo Europeu de Estudo das Espondiloartropatias ${ }^{(3)}(\mathbf{D})$ (Tabela 1), mas não os critérios diagnósticos para uma doença definida dentro do grupo, podem ser considerados EI.

Foram reavaliados recentemente os critérios para dor lombar inflamatória. Foram propostos quatro critérios: dor lombar que melhora com o exercício, mas não com o repouso; despertar noturno em virtude da dor lombar na segunda metade da noite; dor em nádega, alternante; rigidez matinal superior a 30 minutos. É necessária a presença de, pelo menos, dois dos quatro critérios, apresentando uma sensibilidade de $70,3 \%$ e uma especificidade de $81,2 \%$; a presença de um terceiro critério triplica a chance de a dor lombar ser realmente inflamatória ${ }^{(33)}(\mathbf{D})$.

Também se propôs um algoritmo para classificar os pacientes como espondiloartropatia axial precoce ${ }^{(34)}(\mathbf{D})$; a necessidade de se solicitar o HLA-B27 e, em muitas situações, ressonância nuclear magnética de esqueleto axial dificulta sua aplicação na rotina diária de consultório médico no Brasil. Recentemente, descreveram-se padrões de inflamação aguda ${ }^{(35)}(\mathbf{D})$ e alterações $\operatorname{crônicas~}^{\left({ }^{(36)}\right.}(\mathbf{D})$ na ressonância nuclear magnética do esqueleto axial de pacientes com EA, geralmente na sequência STIR (short tau inversion recovery).

\section{TRATAMENTO}

O tratamento das EI é o mesmo das doenças definidas dentro do grupo, enfatizando-se que as formas indiferenciadas costumam ter curso evolutivo mais leve e com menos complicações que as outras doenças do grupo. O uso de infliximabe $^{(37)}(\mathbf{C})$ e etanercepte ${ }^{(38)}(\mathbf{C})$ apresenta resultados promissores nas EI.

\section{UVEÍTE ANTERIOR}

As uveítes anteriores, não granulomatosas, com crises agudas geralmente unilaterais e recorrentes, são encontradas 
com freqüência dentro do grupo das espondiloartropatias. Estas uveítes anteriores agudas (UAA) podem ocorrer em até $40 \%$ dos pacientes com EA, sendo menos freqüentes nas outras doenças do $\operatorname{grupo}^{(39)}(\mathbf{D})$. Podem representar o primeiro sintoma clínico de uma espondiloartropatia indiferenciada ${ }^{(40)}(\mathbf{C})$.

Nas formas crônicas, recidivantes, pode-se encontrar alterações no segmento posterior, tais como vitreíte, edema de mácula e edema do disco óptico ${ }^{(41)}(\mathbf{D})$. Estudos avaliando o prognóstico oftalmológico da UAA associada à presença do HLA-B27 apresentam resultados contraditórios, estando associados a bom prognóstico ${ }^{(42)}$ (A), mau prognóstico ${ }^{(43)}(\mathbf{A})$ ou nenhuma diferença ${ }^{(44)}(\mathbf{B})$. Considerando-se que a UAA HLA-B27 positiva apresenta alta frequiência de recorrências ${ }^{(45)}(\mathbf{C})$ e que as sucessivas recorrências estão associadas a um pior prognóstico ocular ${ }^{(46)}$ (C), pode-se inferir que o HLA-B27 tende a estar associado a um significativo impacto na deficiência visual ${ }^{(47)}(\mathbf{C})$. As UAA associadas ao HLA-B27 são mais freqüentes em homens e tendem a aparecer em indivíduos mais jovens ${ }^{(43)}$ (C), estando associadas à maior possibilidade de desenvolver sintomas articulares ${ }^{(42)}(\mathbf{B})$ e evoluir para $\mathrm{EA}^{(48)}(\mathbf{C})$.

\section{TRATAMENTO}

Quando um paciente com espondiloartropatia apresenta uma crise aguda de uveíte anterior, deve-se encaminhálo a um oftalmologista para confirmar o diagnóstico. $\mathrm{Na}$ confirmação da crise de uveíte anterior, o oftalmologista indicará o uso de corticosteróide tópico (para diminuir a inflamação) e midriáticos (para dilatar a pupila); deve-se reservar o uso de corticoterapia sistêmica aos casos graves com complicações e àqueles com recorrência das crises, especialmente se a doença é bilateral ${ }^{(49)}(\mathbf{D})$, onde a descontinuação da corticoterapia oral deverá ser feita de modo mais lento ${ }^{(49)}(\mathbf{D})$.

$\mathrm{O}$ uso da sulfasalazina parece diminuir o número e a intensidade das crises de uveíte anterior nos pacientes com $\mathrm{EA}^{(50)}(\mathbf{A})$, embora esta afirmação seja controversa entre os oftalmologistas. Nos raros casos de uveíte crônica, pode-se indicar o uso de drogas imunossupressoras como a azatioprina, o metotrexato e a ciclosporina, em doses convencionais, isoladas ou associadas, em algumas situações (necessidade de dose de manutenção de corticosteróide elevada por tempo prolongado, contra-indicação ao uso do corticosteróide, inflamação crônica refratária, acometimento de pólo posterior $)^{(49)}(\mathbf{D})$. O uso dos antibióticos por longo prazo nas uveítes anteriores não mostrou resultados satisfatórios ${ }^{(51)}(\mathbf{A})$.

Pode-se indicar o uso dos agentes biológicos antiTNF $\alpha$ nos casos de UAA HLA-B27 positivo resistentes ao tratamento convencional e/ou com risco potencial de perda visual. Tem-se indicado o infliximabe nas uveítes recorrentes ou crônicas, complicadas com edema cistóide de mácula e resistentes à terapia convencional com corticoterapia oral e/ou imunossupressores ${ }^{(52)}(\mathbf{B})$. O infliximabe parece ser especialmente eficaz nos casos de UAA HLAB27 positivo associada a manifestações extra-oculares, como as articulares ${ }^{(53)}(\mathbf{B})$. O infliximabe tem sido efetivo no tratamento de uveítes anteriores enquanto está sendo administrado; não produz remissão das crises de uveíte e recidivas ocorrem após algum tempo da sua suspensão. $\mathrm{O}$ uso do etanercepte nas uveítes anteriores apresenta resultados controversos, podendo estar associado à remissão ${ }^{(54)}(\mathbf{B})$ e à indução de casos de uveíte ${ }^{(55)}(\mathbf{B})$, denotando que sua real eficácia necessita ser melhor estabelecida em estudos controles de longo prazo.

\section{ESPONDILOARTROPATIAS JUVENIS}

As espondiloartropatias juvenis (que se iniciam em indivíduos menores de 16 anos) costumam ter características clínicas que permitem diferenciá-las da doença que se inicia no adulto. Primordialmente, apresentam franco predomínio de acometimento de articulações periféricas, geralmente como uma oligoartrite de articulações de membros inferiores, associada à entesopatia periférica; o acometimento axial costuma ser tardio, muitas vezes já na fase adulta ${ }^{(56)}(\mathbf{D})$.

É comum o diagnóstico de espondiloartropatia indiferenciada, como a Síndrome SEA, caracterizada por uma artrite com entesopatia periférica, soronegativa para o FAN e fator reumatóide, que, em número significativo de casos, costuma evoluir para $\mathrm{EA}^{(57)}(\mathbf{B})$.

\section{TRATAMENTO}

Recomenda-se o uso de $A I N H$ no início do tratamento, em doses habituais, enquanto houver atividade de doença; não existe um AINH específico que seja comprovadamente mais eficaz que os demais ${ }^{(58)}(\mathbf{D})$. O uso de corticosteróides costuma ser indicado somente nos casos potencialmente incapacitantes e não responsivos ao AINH, podendo ser utilizado por via oral ou intra-articular (nos casos de monoartrite persistente $)^{(56)}(\mathbf{B})$. 
A sulfasalazina, na dose de 30 a $50 \mathrm{mg} / \mathrm{kg} /$ dia, apresenta bons resultados no componente periférico da doença ${ }^{(59)}(\mathbf{B})$. O metotrexato e a ciclosporina (isolados ou associados) podem ser eficazes na artrite idiopática juvenil, mas ainda carecem de estudos controle nas espondiloartropatias juvenis ${ }^{(56)}(\mathbf{D})$.

\section{REFERÊNCIAS}

1. Moll JHM, Haslock I, MacRae I, et al.: Associations between ankylosing spondylitis, psoriatic arthritis, Reiter's disease, the intestinal arthropathies, and Behcet's syndrome. Medicine 53:343-64, 1974.

2. Braun J, Sieper J: Building consensus on nomenclature and disease classification for ankylosing spondylitis: results and discussion of a questionnaire prepared for the International Workshop on New Treatment Strategies in Ankylosing Spondylitis. Ann Rheum Dis 61(Supl. III):61-7, 2002.

3. Dougados M, van der Linden S, Juhlin R, et al.: The European Spondylarthropathy Study Group preliminary criteria for the classification of spondylarthropathy. Arthritis Rheum 34:1218-27, 1991.

4. Hamdulay SS, Glynne SJ, Keat: When is arthritis reactive? Postgrad Med J 82:446-53, 2006.

5. Toivanen A, Toivanen P: Reactive arthritis. Best Pract Res Clin Rheumatol 18:689-703, 2004.

6. Zeidler H, Kuipers JG, Köhler L: Chlamydia-induced arthritis. Curr Opin Rheumatol 16:380-92, 2004.

7. Gaston JSH, Lillicrap MS: Arthritis associated with enteric infection. Best Pract Res Clin Rheumatol 17:219-39, 2003.

8. Granfors K: Do bacterial antigens cause reactive arthritis? Rheum Dis Clin North Am 18:37-48, 1992.

9. Rihl M, Kohler L, Klos A, Zeidler H: Persistence infection of Chlamydia in reactive arthritis. Ann Rheum Dis 65:281-4, 2006.

10. Amor B: Reiter's syndrome: diagnosis and clinical features. Rheum Dis Clin North Am 24:677-95, 1998.

11. Kingsley G, Sieper J: Third International Workshop on Reactive Arthritis, 23-26 September 1995, Berlin, Germany. Ann Rheum Dis 55:564-84, 1996.

12. Palazzi C, Olivieri I, D'Amico E, Pennese E, Petricca A: Management of reactive arthritis. Expert Opin Pharmacother 51:61-70, 2004.

13. Clegg DO, Reda DJ, Weisman MH, et al.: Comparison of sulfasalazine and placebo in the treatment of reactive arthritis (Reiter's syndrome). Arthritis Rheum 39:2021-7, 1996.

14. Lally EV, Ho Jr. G: A review of methotrexate therapy in Reiter syndrome. Semin Arthritis Rheum 15:139-45, 1985.

15. Kaipiainen-Seppänen $\mathrm{O}$, Niinisalo H, Korpilähde T, Virolainen $\mathrm{J}$ : Treatment of reactive arthritis with infliximab. Scand J Rheumatol 32:122-4, 2003.

16. Flagg SD, Meador R, et al.: Decreased pain and synovial inflammation after etanercept therapy in patients with reactive and undifferentiated arthritis: an open label trial. Arthritis Care Res 53:613-7, 2005.

17. Sieper J, Braun J: Treatment of reactive arthritis with antibiotics. Br J Rheumatol 37:717-20, 1998.

18. Sieper J, Fendler C, Laitko S, et al.: No benefit of long-term ciprofloxacin treatment in patients with reactive arthritis and undifferentiated oligoarthritis. Arthritis Rheum 42:1386-96, 1999.
Quanto aos agentes biológicos, infliximabe e etanercepte, estudos abertos e com casuísticas pequenas mostram resultados favoráveis em uma proporção significativa dos pacientes $^{(60)}(\mathbf{D})$. É importante lembrar que as vacinas de vírus vivos são contraindicadas em pacientes em tratamento com imunossupressores ou agentes biológicos.

19. Yli-Kertula T, Luukkainen R, Yli-Kertula U, et al.: Effect of a three month course of ciprofloxacin on the late prognosis of reactive arthritis. Ann Rheum Dis 62:880-4, 2003.

20. Smieja M, MacPherson DW, Kean W, et al.: Randomised, doubleblinded, placebo-controlled trial of doxycicline for chronic seronegative arthritis. Ann Rheum Dis 60:1088-94, 2001.

21. Carter JD, Valeriano J, Vasey FB: Doxycicline versus doxycicline and rifampin in undifferentiated spondyloarthropathy, with special reference to Chlamydia-induced arthritis. A prospective, randomized 9-month comparison. J Rheumatol 31:1973-80, 2004.

22. Holden W, Orchard T, Wordsworth P: Enteropathic arthritis. Rheum Dis Clin North Am 29:513-30, 2003.

23. Smale S, Natt RS, Orchard TR, Russell AS, Bjarnason I: Inflammatory bowel disease and spondylarthropathy. Arthritis Rheum 44:2728-36, 2001.

24. Baumgart DC, Sandborn WJ: Inflammatory bowel disease: clinical aspects and established and evolving therapies. Lancet 369:1641-57, 2007.

25. Hanauer SB, Stromberg U: Oral pentasa in the treatment of active Crohn's disease: a meta-analysis of double-blind, placebocontrolled trials. Clin Gastroenterol Hepatol 2:379-88, 2004.

26. Feagan BG, Rochon J, Fedorak RN, et al.: Methotrexate for the treatment of Crohn's disease. N Engl J Med 332:292-7, 1995.

27. Bouhnik Y, Lemman M, Mary JY, et al.: Long-term follow-up of patients with Crohn's disease treated with azathioprine or 6-mercaptopurine. Lancet 347:215-9, 1996.

28. Hanauer SB, Feagan BG, Lichtenstein GR, et al.: Maintenance infliximab for Crohn's disease: the ACCENT 1 randomized trial. Lancet 2002;359:1541-9.

29. Colombel JF, Sandborn WJ, Rutgeerst P, et al.: Adalimumab for maintenance of clinical response and remission in patients with Crohn's disease: the CHARM trial. Gastroenterology 132:52-65, 2007.

30. Zeidler H, Mau W, Khan MA: Undifferentiated spondyloarthropathies. Rheum Dis Clin North Am 18:187-202, 1992.

31. Mau W, Zeidler $H$, Mau R, et al.: Clinical features and prognosis of patients with possible ankylosing spondylitis. Results of a 10year follow-up. J Rheumatol 15:1109-14, 1988.

32. Sampaio-Barros PD, Conde RA, Donadi EA, et al.: Undifferentiated spondyloarthropathies in Brazilians: importance of HLA-B27 and the B7-CREG alleles in characterization and disease duration. J Rheumatol 30:2632-7, 2003.

33. Rudwaleit M, Metter A, Listing J, Sieper J, Braun J: Inflammatory back pain in ankylosing spondylitis: a reassessment of the clinical history for application as classification and diagnostic criteria. Arthritis Rheum 54:569-78, 2006.

34. Rudwaleit M, van der Heijde D, Khan MA, Braun J, Sieper J: How to diagnose axial spondyloarthritis early. Ann Rheum Dis 63:535-43, 2004. 
35. Baraliakos X, Landewé R, Hermann K-G, et al.: Inflammation in ankylosing spondylitis: a systematic description of the extent and frequency of acute spinal changes using magnetic resonance imaging. Ann Rheum Dis 64:730-4, 2005.

36. Braun J, Baraliakos X, Golder W, et al.: Analysing chronic spinal changes in ankylosing spondylitis: a systematic comparison of conventional $\mathrm{x}$-rays with magnetic resonance imaging using established and new scoring systems. Ann Rheum Dis 63:1046$55,2004$.

37. Brandt J, Haibel H, Reddig J, Sieper J, Braun J: Successful treatment of severe undifferentiated spondyloarthropathy with the anti-tumor necrosis factor- $\alpha$ monoclonal antibody infliximab. J Rheumatol 29:118-22, 2002.

38. Brandt J, Khariouzov A, Listing J, et al.: Successful short term treatment of patients with severe undifferentiated spondyloarthritis with the anti-tumor necrosis factor- $\alpha$ fusion receptor protein etanercept. J Rheumatol 31:531-8, 2004.

39. Bañares A, Hernandez-Garcia C, Fernandez-Gutierrez B, Jover JJ: Eye involvement in the spondyloarthropathies. Rheum Dis Clin North Am 24:771-784, 1998.

40. Pato E, Bañares A, Jover JJ, et al.: Undiagnosed spondyloarthropathy in patients presenting with anterior uveitis. J Rheumatol 27:2198-202, 2000.

41. Oréfice F, Carvalho MAP, Moreira C: Espondiloartropatias. In: Oréfice F (ed.). Uveíte clínica e cirúrgica: texto e atlas. 2. ed. Rio de Janeiro: Cultura Médica, 2004.

42. Linssen A, Meenken C: Outcomes of HLA-B27-positive and HLA-B27-negative acute anterior uveitis. Am J Ophthalmol 120:351-61, 1995.

43. Monnet D, Breban M, Hudry C, Dougados M, Brezin AP: Ophthalmic findings and frequency of extraocular manifestations in patients with HLA-B27 uveitis: a study of 175 cases. Ophthalmology 111:802-9, 2004.

44. Rothova A, Suttorp-van Schulten MS, Frits Treffers W, Kijlstra A: Causes and frequency of blindness in patients with intraocular inflammatory disease. Br J Ophthalmol 80:332-6, 1996.

45. Sampaio-Barros PD, Conde RA, Bonfiglioli R, Bértolo MB, Samara AM: Characterization and outcome of uveitis in 350 patients with spondyloarthropathies. Rheumatol Int 26:1143-46, 2006.

46. Wakefield D, Easter J, Penny R: Clinical features of HLA-B27 anterior uveitis. Aust J Ophthalmol 12:191-6, 1984.

47. Power WJ, Rodriguez A, Pedroza-Seres M, Foster CS: Outcomes in anterior uveitis associated with the HLA-B27 haplotype. Ophthalmology 105:1646-51, 1998.
48. Carvalho MAP, Campos WR, Araújo CAA, Lacerda RR, Oréfice F: Uveítes anteriores não granulomatosas, espondiloartropatias e HLA-B27. Rev Bras Reumatol 39:195-202, 1999.

49. Jabs DA, Rosenbaum JT, Foster CS, et al.: Perspective guidelines for the use of immunosuppressive drugs in patients with ocular inflammatory disorders: recommendations of an expert panel. Am J Ophtahlmol 30:492-513, 2000.

50. Benitez-del-Castilho JM, Garcia-Sanchez J, Iradier T, Bañares A: Sulfasalazine in the prevention of anterior uveitis associated with ankylosing spondylitis. Eye 14:340-3, 2000.

51. Wakefield D, McCluskey P, Verma M, et al.: Ciprofloxacin treatment does not influence course or relapse rate of reactive arthritis and anterior uveitis. Arthritis Rheum 42:1894-7, 1999.

52. Suhler EB, Smith JR, Wertheim MS, et al.: A prospective trial of infliximab therapy or refractory uveitis. Preliminary safety and efficacy outcomes. Arch Ophthalmol 123:903-12, 2005.

53. Guignard S, Gossec L, Salliot C, et al.: Efficacy of tumor necrosis factor blockers in reducing uveitis flares in patients with spondylarthropathy: a retrospective study. Ann Rheum Dis 65:1631-4, 2006.

54. Braun J, Baraliakos X, Listing J, Sieper J: Decreased incidence of anterior uveitis in patients with ankylosing spondylitis treated with the anti-tumor necrosis factor agents infliximab and etanercept. Arthritis Rheum 52:2447-51, 2005.

55. Taban M, Dupps WJ, Mandell B, Perez VL: Etanerceptassociated inflammatory eye disease: case report and review of the literature. Ocul Immunol Inflamm 14:145-50, 2006.

56. Burgos-Vargas R, Pacheco-Tena C, Vazquez-Mellado J: Juvenileonset spondyloarthropathies. Rheum Dis Clin North Am 23:569-98, 1997.

57. Cabral DA, Oen KG, Petty RE: SEA syndrome revisited: a longterm followup of children with a syndrome of seronegative enthesopathy and arthropathy. J Rheumatol 19:1282-5, 1992.

58. Huang F, Zhang J, Zhu J, Guo J, Yang C: Juvenile spondyloarthropathies: the Chinese experience. Rheum Dis Clin North Am 29:531-547, 2003.

59. Burgos-Vargas R, Vazquez-Mellado J, Pacheco-Tena C, Hernandez-Garduno A, Goycochea-Robles MV: A 26 week, randomized, double-blind, placebo controlled exploratory study of sulfasalazine in juvenile-onset spondyloarthropathies. Ann Rheum Dis 61:941-2, 2002.

60. Henrickson M, Reiff A: Prolonged efficacy of etanercept in refractory enthesitis-related arthritis. J Rheumatol 31:2055-61, 2004. 